\title{
Non-Invasive Low Pulsed Electrical Fields for Inducing BBB Disruption in Mice-Feasibility Demonstration
}

\author{
Shirley Sharabi ${ }^{1, *(\mathbb{D}}$, David Last ${ }^{1}$, Dianne Daniels ${ }^{1}$, Ido Didi Fabian ${ }^{2,3}{ }^{\mathbb{C}}$, Dana Atrakchi ${ }^{4}$, Yael Bresler ${ }^{1,3,4}$, \\ Sigal Liraz-Zaltsman ${ }^{4,5,6}$, Itzik Cooper ${ }^{4,7}$ and Yael Mardor ${ }^{1,3}$ (D) \\ 1 The Advanced Technology Center, Sheba Medical Center, Tel Hashomer, Ramat-Gan 5262000, Israel; \\ david.last@sheba.health.gov.il (D.L.); Dianne.daniels@sheba.health.gov.il (D.D.); \\ yael.bresler@sheba.health.gov.il (Y.B.); yael.mardor@sheba.health.gov.il (Y.M.) \\ 2 Goldschleger Eye Institute, Sheba Medical Center, Tel Hashomer, Ramat-Gan 5262000, Israel; \\ didi@didifabian.com \\ 3 Sackler School of Medicine, Tel-Aviv University, Tel-Aviv 69978, Israel \\ 4 The Joseph Sagol Neuroscience Center, Sheba Medical Center, Tel Hashomer, Ramat Gan 52621, Israel; \\ Dana.Atrakchi@sheba.health.gov.il (D.A.); Sigal.LirazZaltsman@sheba.health.gov.il (S.L.-Z.); \\ itzik.cooper@sheba.health.gov.il (I.C.) \\ 5 Department of Pharmacology, Institute for Drug Research, Hebrew University, Jerusalem 9112001, Israel \\ 6 Institute for Health and Medical Professions, Department of Sports Therapy, Ono Academic College, \\ Kiryat Ono 5545173, Israel \\ 7 Interdisciplinary Center Herzliya, Herzliya 4610101, Israel \\ * Correspondence: shirley.sharabi@sheba.health.gov.il; Tel.: +972-3-530-5808; Fax: +972-3-530-3146
}

Citation: Sharabi, S.; Last, D.;

Daniels, D.; Fabian, I.D.; Atrakchi, D.;

Bresler, Y.; Liraz-Zaltsman, S.; Cooper,

I.; Mardor, Y. Non-Invasive Low

Pulsed Electrical Fields for Inducing

BBB Disruption in Mice-Feasibility

Demonstration. Pharmaceutics 2021,

13, 169. https://doi.org/10.3390/

pharmaceutics13020169

Academic Editor: Xavier Declèves

Received: 26 November 2020

Accepted: 23 January 2021

Published: 27 January 2021

Publisher's Note: MDPI stays neutral with regard to jurisdictional claims in published maps and institutional affiliations.

Copyright: (C) 2021 by the authors. Licensee MDPI, Basel, Switzerland. This article is an open access article distributed under the terms and conditions of the Creative Commons Attribution (CC BY) license (https:/ / creativecommons.org/licenses/by/ $4.0 /)$.

\begin{abstract}
The blood-brain barrier (BBB) is a major hurdle for the treatment of central nervous system disorders, limiting passage of both small and large therapeutic agents from the blood stream into the brain. Thus, means for inducing BBB disruption (BBBd) are urgently needed. Here, we studied the application of low pulsed electrical fields (PEFs) for inducing BBBd in mice. Mice were treated by low PEFs using electrodes pressed against both sides of the skull (100-400 square $50 \mu$ s pulses at $4 \mathrm{~Hz}$ with different voltages). BBBd as a function of treatment parameters was evaluated using MRI-based treatment response assessment maps (TRAMs) and Evans blue extravasation. A 3D numerical model of the mouse brain and electrodes was constructed using finite element software, simulating the electric fields distribution in the brain and ensuring no significant temperature elevation. BBBd was demonstrated immediately after treatment and significant linear regressions were found between treatment parameters and the extent of BBBd. The maximal induced electric field in the mice brains, calculated by the numerical model, ranged between 62.4 and $187.2 \mathrm{~V} / \mathrm{cm}$ for the minimal and maximal applied voltages. These results demonstrate the feasibility of inducing significant BBBd using non-invasive low PEFs, well below the threshold for electroporation.
\end{abstract}

Keywords: blood-brain barrier disruption; pulsed electrical fields; MRI; treatment response assessment maps; non-invasive; neurodegenerative diseases

\section{Introduction}

Despite the rise in the prevalence of neurodegenerative diseases in recent years, mainly due to the increasing lifespan, drug development for central nervous system (CNS) disorders such as stroke, brain tumors and neurodegenerative disorders such as Alzheimer's disease, amyotrophic lateral sclerosis and Parkinson's disease is extremely challenging. The reasons include brain complexity, side effects and the blood-brain barrier (BBB) which prevents most therapeutics from reaching the CNS [1].

The BBB is a multicellular barrier composed of brain endothelial cells as well as pericytes and astrocytes. The brain microvasculature endothelial cells have highly developed tight junctions and adherent junctions complexes which together with a wide range of efflux pumps limit transport of up to $98 \%$ of small molecules and almost $100 \%$ of large 
molecules form the blood to the brain tissue [2]. Although essential for normal brain function, the BBB is a significant obstacle for the treatment of brain diseases as it limits the passage of most therapeutic agents from the blood stream into brain tissue.

Research for CNS drug delivery focuses on three main approaches [3]: (1) reformulating existing drugs to cross the BBB by physiological transport mechanisms and drugdelivery approaches such as microspheres and colloidal drug-carriers [4]; (2) minimally invasive/invasive local delivery methods such as convection-enhanced delivery $[5,6]$ and biodegradable wafers; and (3) disrupting the $\mathrm{BBB}$, where non-localized methods include osmotic BBB disruption (BBBd) [7], while localized approaches include laser interstitial thermotherapy, MRI-guided focused ultrasound (MRgFUS) [8] and electroporation (EP) [9].

During EP, high pulsed electrical fields (PEFs), in the order of hundreds to thousands volts per centimeter $(\mathrm{V} / \mathrm{cm})$, are applied using at least two electrodes, destabilizing the cell membrane and inducing nano-scale pores in the cell's membranes. When the electric fields are applied such that the pores can reseal within few minutes, the treatment is termed reversible EP and is used to increase the uptake of therapeutic molecules. When cell membranes are permeabilized in a manner leading to cellular death [10], it is called irreversible EP. Both reversible and irreversible EP are methods in different stages of development for treating tumors outside and inside the CNS [11-16].

It has been recently demonstrated that EP can induce transient BBBd in vivo, thus enhancing intraparenchymal drug uptake. Such treatments showed improved survival and reduced tumor volume or growth rates in glioma-bearing rats and dogs [17-20]. The electric field threshold for inducing BBBd by EP was shown to be $500-700 \mathrm{~V} / \mathrm{cm}$ depending on treatment parameters $[17,19]$. Recently, Lorenzo et al. [21] demonstrated that highfrequency EP (H-FRE, delivery of high numbers of short bipolar pulses at $250-500 \mathrm{kHz}$ ) also induces BBBd. The treatment was conducted using two needle electrodes inserted into the brain parenchyma with a voltage to distance ratio of $600 \mathrm{~V} / \mathrm{cm}$. The authors determined the threshold for BBBd to be $113 \mathrm{~V} / \mathrm{cm}$. These treatments, although showing minimal side effects, require at least one intracranial electrode and often require craniotomy. Moreover, when EP is used for inducing large BBBd volumes, irreversible EP may occur, resulting in brain damage $[17,18,22]$.

We recently introduced the application of low PEFs, in the order of $15-100 \mathrm{~V} / \mathrm{cm}$, for inducing BBBd in vitro well below the threshold for EP [23]. Unlike EP, which induces transcellular BBBd, this method was found to induce paracellular BBBd.

In the current study, we aimed to demonstrate the feasibility of applying non-invasive (intact skull) low PEFs for inducing BBBd in naïve mice. A quantification method using MRI-based treatment response assessment maps (TRAMs) [24,25] was developed. The TRAMs were applied in order to quantify the effects of treatment protocols on the volume and intensity of BBBd [20].

\section{Methods}

\subsection{Animals}

The study was approved by the Animal Care and Use Committee of Sheba Medical Center and was performed in accordance with the guidelines of ARRIVE.

Sixty-four Hsd mice, weighting 25-30 g, were used in this study. A 12:12-h lightdark cycle was maintained. Food and water were provided ad libitum. Fifty-eight mice underwent PEFs treatment followed by delayed contrast MRI and euthanasia or Evans blue (EB) infusion followed by delayed perfusion and brain extraction, for BBBd assessments. An additional six mice underwent PEFs treatment and were kept for observation. The mice were scanned by MRI $48 \mathrm{~h}$ post-treatment for assessment of treatment-related toxicity.

\subsection{MRI Experimental Outline}

Anesthesia was administered by intramuscular injections of $250 \mu \mathrm{L}$ of $1 \mathrm{~mL} / \mathrm{kg}$ ketamine and $0.5 \mathrm{~mL} / \mathrm{kg}$ xylazine. The anesthetized mice were subjected to a midline scalp incision and the skin was separated from both sides to expose the skull. Two stainless steel 
$1.5 \mathrm{~cm}$ square plate electrodes (Caliper Electrode, Harvard Apparatus, Holliston, MA, USA) were pressed against the sides of the intact skull after application of conductive gel. The distance between the electrodes was $1.2-1.3 \mathrm{~cm}$.

The mice were treated by 100-400 square pulses with a pulse duration of $50 \mu$ s at 100-300 V. The pulses were applied in sets of 25 pulses at a frequency of $4 \mathrm{~Hz}$ with 5-s intervals between sets. Sham procedures for the control rats included anesthesia, skin incision, placing the electrodes and leaving the electrodes in place for $60 \mathrm{~s}$. At 2-5 min post-PEFs application, the mice were scanned by MRI to evaluate BBBd. The MRI contrast agent was injected into the tail vein in the MRI, immediately prior to the first scan.

The MRI experiments were designed to demonstrate the feasibility of inducing BBBd using non-invasive low PEFs and to study the dependency on the treatment parameters. Three to six mice were treated in each treatment group (100 V with 100, 200, 300 and 400 pulses; $150 \mathrm{~V}$ with 100, 200, 300 and 400 pulses; 100 pulses with 100, 150, 200 and $300 \mathrm{~V})$, up to a total of 43 mice.

Six mice were divided into two treatment groups of 3 mice each (100 V with 100 pulses and $300 \mathrm{~V}$ with 100 pulses) and scanned by MRI $48 \mathrm{~h}$ post-treatment to evaluate possible damage.

\subsection{MRI Data Acquisition}

The MRI experiments were performed using a GE Optima MRI (1.5T) with an 8channel phased array wrist coil. The contrast agent (Gd-DOTA, $0.016 \mathrm{mmol} / \mathrm{kg}$, Dotarem, Guerbert) was injected into the tail vein immediately prior to the scan. The mice were scanned with the following MR sequences: repeated 3D contrast-enhanced T1-weighted MRI (T1-MRI) for at least 30 min after contrast injection to depict BBBd, T2-weighted MRI (T2-MRI) to depict edema or damage and T2*-weighted gradient echo (GE) and susceptibility-weighted MRI (SWAN) to depict potential bleeding. MRI sequences were acquired with the following parameters: 3D Cube T1-MRI: $10 \mathrm{~cm}$ FOV (phase FOV: 0.8), $244.14 \mathrm{kHz}$ bandwidth, TE/TR = 21.4/602 ms, $0.8 \mathrm{~mm}$ slice thickness, 2562 matrix size, resulting in a voxel size of $0.39 \times 0.39 \times 0.4 \mathrm{~mm}^{3}$ after zerofill; $2 \mathrm{D}$ fast spin echo T2-MRI: $12 \mathrm{~cm}$ FOV (phase FOV: 0.5), $1.0 \mathrm{~mm}$ slice thickness, $20.83 \mathrm{kHz}$ bandwidth, TE/TR = 85/5300 ms, $256 \times 224$ matrix size, resulting in a voxel size of $0.43 \mathrm{~mm}^{3}$ after zerofill. GE images were acquired with a $256 \times 224$ matrix, $12 \mathrm{~cm}$ FOV, TE/TR $=15 / 300 \mathrm{~ms}, \mathrm{BW}=15.63 \mathrm{kHz}, \mathrm{NEX}=2$, a flip angle of $15^{\circ}$ and $1 \mathrm{~mm}$ slice thickness. The in-plane voxel size was $\left(0.47 \mathrm{~mm}^{2}\right) .3 \mathrm{D}$ SWAN: $10 \mathrm{~cm}$ FOV (phase FOV: 0.8), $1.0 \mathrm{~mm}$ slice thickness, $31.25 \mathrm{kHz}$ bandwidth, TE/TR $=47 / 124 \mathrm{~ms}, 30$ degrees flip angle, $320 \times 256$ matrix size, resulting in a voxel size of $0.43 \mathrm{~mm}^{3}$ after zerofill.

\subsection{Image Analysis}

For each mouse, TRAMs were calculated for visualization of subtle BBBd using Matlab (R2014a, Mathworks, Natick, MA, USA) as previously described [20]. In short, all T1-MRI series were registered to the 1st post-contrast T1-MRI series using 3D rigid registration. The 1st series post-contrast was than subtracted from each of the delayed series generating TRAMs. In the TRAMs, contrast clearance, resulting in a negative signal, was depicted blue and contrast accumulation, resulting in a positive signal, was depicted red. No change was depicted green (Figure 1B).

For quantification of BBBd volumes and intensities, a pixel by pixel analysis was conducted. First, a region of interest (ROI) was plotted over all the brain slices so that the entire brain was segmented for each mouse (Figure 1A) for all time points post-contrast injection. Next, within the brain region, the intensity of each pixel was plotted as a function of time post-contrast injection and fitted to a 2-exponential function, based on a twocompartment exchange model of Tofts et al. [26-28]. The fit resulted in quantification of the following parameters: time to peak, maximal intensity increase, wash-in and wash-out slopes and coefficient of determination $\left(\mathrm{r}^{2}\right)$. Then, maps of the maximal intensity increase and $r^{2}$ were generated (Figure 1C,D). Pixels presenting with BBBd were defined as those 
above a threshold of 1.05 for the intensity increase (representing a $>5 \%$ increase in signal intensity) and 0.6 for $\mathrm{r}^{2}$.

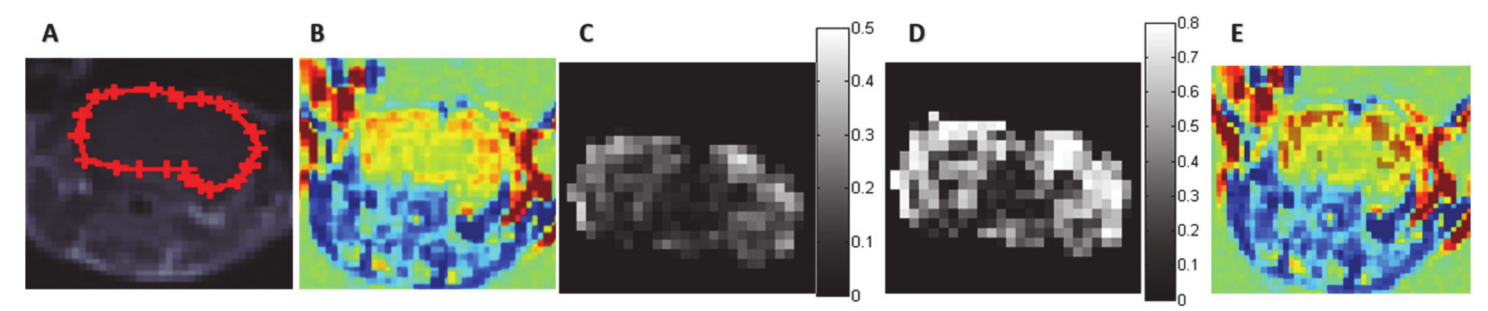

Figure 1. Examples of calculated maps: (A) A T1-MRI slice with the plotted brain region of interest (ROI) overlaid. (B) Treatment response assessment maps (TRAMs) calculated by subtracting the 1st series post-contrast from the series obtained $30 \mathrm{~min}$ post-contrast injection showing red (contrast accumulation) regions (demonstrating higher blood-brain barrier disruption (BBBd) in the cortex). (C) Maximal intensity increase map in the same brain slice. (D) $\mathrm{r}^{2}$ map depicting the fit $\mathrm{r}^{2}$ of each pixel in the same brain slice. (E) Pixels included in the BBBd ROI (intensity increase $>5 \%$ and $\mathrm{r}^{2}>0.6$ ), marked in brown and overlaid on the TRAMs.

Pixels above these thresholds were included in the BBBd ROI (Figure 1E). Lastly, a cluster analysis was conducted, removing clusters of less than 10 pixels $\left(0.6 \mathrm{~mm}^{3}\right)$ from the BBBd ROI in order to minimize inclusion of noise. BBBd volume was then calculated by multiplying the number of pixels in the BBBd ROI by the pixel volume. BBBd intensity was calculated as the mean maximal intensity increase in the BBBd ROI.

\subsection{Evans Blue Experimental Outline}

EB extravasation is the most widely used method to detect BBBd. EB is limited by the $\mathrm{BBB}$; therefore, EB in brain tissue is indicative of BBB permeability changes [29]. The EB experiments were designed to quantify the amount of EB that crosses the BBB in specific brain regions. Fifteen mice were treated with low PEFs in 3 treatment groups of 5 mice each: sham, $100 \mathrm{~V}$ with 100 pulses and $200 \mathrm{~V}$ with 100 pulses.

The surgical procedure and PEFs treatments were performed similarly to those of the MRI experiments. The experiments were performed using the optimized protocol of Wang et al. [30]. In short, a $2 \%$ EB solution $(4 \mathrm{~mL} / \mathrm{kg})$ was injected into 15 mice tails immediately post-PEFs application and was allowed to circulate for $20 \mathrm{~min}$, after which the mice were thoroughly perfused for $8 \mathrm{~min}$ with $60 \mathrm{~mL}$ of saline. Following perfusion, brains were dissected (cortex, cerebellum and rest of the brain), weighed in Eppendorf tubes and frozen a $-80{ }^{\circ} \mathrm{C}$. Frozen brain samples were thawed and mixed with a 1:3 $(\mathrm{W}(\mathrm{mg}) / \mathrm{V}(\mu \mathrm{L}))$ ratio of cold $50 \%$ trichloroacetic acid (TCA) in $0.9 \%$ saline. Samples were homogenized for $5 \mathrm{~min}$ (continuous beating) using a pre-cooled metal-bead homogenizer (BULLET BLENDER ${ }^{\circledR}$ BBX24). Tubes were then centrifuged for $20 \mathrm{~min}$ at $10,000 \times g$ at $4{ }^{\circ} \mathrm{C}$. An amount of $30 \mu \mathrm{L}$ was collected from each sample in duplicates into a clear 96-well plate. An amount of $90 \mu \mathrm{L}$ of $95 \%$ ethanol was then added to each sample and spectroscopically detected by $620 / 680 \mathrm{~nm}$ excitation/emission using a florescence plate reader (Tecan Infinite F200). The amount of EB in each sample was determined from a calibration curve (ranging from 0.083 to $4 \mu \mathrm{g} / \mathrm{mL}$ ) prepared with EB that was dissolved in a 50\% TCA solution (30 $\mu \mathrm{L})$ and $90 \mu \mathrm{L}$ of $95 \%$ ethanol and was then normalized to the brain sample weight.

\subsection{Numerical Modeling}

A 3D finite elements model of a mouse brain and electrodes was constructed using COMSOL (COMSOL Multiphysics 5.3a, Stockholm, Sweden). The mouse head was modeled as an ellipsoid of $6 \times 8 \times 7 \mathrm{~mm}$ semi-axes with the lower seventh removed. The resulting shape had a $12 \mathrm{~mm}$ diameter in the coronal plain, a maximal length of $16 \mathrm{~mm}$ (sagittal plain) and maximal height of $12 \mathrm{~mm}$ (Figure 2A). Additional internal layers were added representing the skull $(0.6 \mathrm{~mm})$, dura $(0.1 \mathrm{~mm})$ and brain. The final brain volume reached $510.4 \mathrm{~mm}^{3}$. These parameters of the head model were obtained by measuring the 
volume and semi-axes of the head and brain of a mouse scanned by MRI. The number of elements in the finalized model was 666,024 and 211,022 in the brain domain. The tissue electrical and thermal properties were obtained from the literature (Table 1).

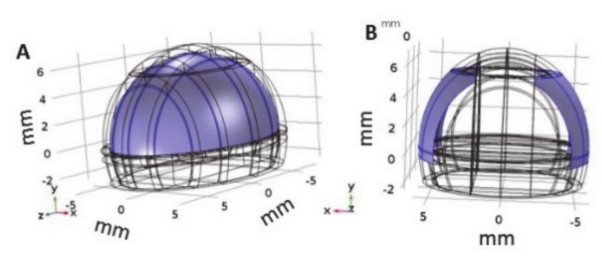

Figure 2. Model geometry and electrodes position (A). Geometry of the mice head model with the brain domain in purple. (B) Location of the electrodes (purple) where the boundary conditions were applied to.

Table 1. Tissue electric and thermal properties.

\begin{tabular}{cccc}
\hline & Brain & Dura & Skull \\
\hline Thermal conductivity W/(m*K) & $0.565[31]$ & $0.44[32]$ & $0.16[31]$ \\
Heat capacity J/(kg*K) & $3650[33]$ & $3364[32]$ & $1700[31]$ \\
Density $\mathrm{Kg} / \mathrm{m}^{3}$ & $1040[31]$ & $1174[32]$ & $1500[31]$ \\
Blood perfusion rate $\mathrm{mL} /\left(\mathrm{s}^{*} \mathrm{~cm}^{3}\right)$ & $0.007[33]$ & $0.143 \times 10^{-3}[31]$ & $0.143 \times 10^{-3}[31]$ \\
Metabolic heat production $\mathrm{W} / \mathrm{m}^{3}$ & $10437[33]$ & $4144[32]$ & $70[31]$ \\
Electric conductivity $\mathrm{s} / \mathrm{m}$ & $0.258[34]$ & $0.06[32]$ & $0.01[35]$ \\
\hline
\end{tabular}

The Laplace Equation for electric potential was used to describe the electric field:

$$
\nabla \cdot(\sigma \nabla \varphi)=0
$$

where $\sigma$ represents the tissue electric conductivity and $\varphi$ is the electric potential. The Dirichlet boundary condition was applied to the surface of the electrodes (Figure 2B) and the Neumann boundary condition was set to zero for the remaining outer surface boundaries as they were considered electrically isolative. The modified Pennes bioheat equation with an additional Joule heating source term was used to determine thermal effects. The outer surface of the model was considered thermally insulted:

$$
\nabla \cdot(k \nabla T)+w_{b} c_{b}\left(T_{a}-T\right)+Q_{m e t}+\sigma|\nabla \varphi|^{2}=\rho c_{p} \frac{\partial T}{\partial t}
$$

where $k$ is the tissue thermal conductivity, $T$ is the temperature, $\mathrm{w}_{\mathrm{b}}$ is the blood perfusion, $c_{b}$ is the blood heat capacity, $T_{a}$ is the arterial temperature, $Q_{\text {met }}$ is the metabolic heat generation, $\rho$ is the tissue density, $c_{p}$ is the heat capacity of the tissue, $\varphi$ is the electrical potential and $\sigma$ is the electrical conductivity. The brain temperature prior to treatment was set to $36.8^{\circ} \mathrm{C}$.

The thermal model was solved using a duty cycle approach [36]. In short, instead of calculating the Joule heating for each pulse and implementing the new temperature as the next step initial temperature, a time-dependent solver was applied, and the thermal dissipation was multiplied by the pulse length $(50 \mu \mathrm{s})$ and the number of pulses. The duty cycle approach does not take into consideration cooling effects during the pulse intervals and thus is considered more conservative in evaluating temperature increase.

\subsection{Statistical Analysis}

Results are presented as means with standard errors. The differences between the sham and the minimal treatment (100 pulses at $100 \mathrm{~V})$ groups were studied by an independent samples $t$ test. The dependency of the extent of BBBd in the number of pulses and treatment voltage was studied using a linear regression analysis [37] both for the MRI 
experiments and the EB experiments. Linearity, homoscedasticity and normality of the residuals were verified.

\section{Results}

\subsection{MRI Experiments}

At 2-5 min following PEFs treatment, the mice were scanned by MRI. T2-MRI, SWAN and repeated contrast-enhanced 3D T1-MRIs were acquired. The TRAMs were generated for each mouse.

No signs of edema, damage or bleeding were observed in any of the treated mice. Standard 3D T1-MRI obtained immediately post-contrast injection showed no clear enhancement in the brain parenchyma in any of the mice (Figure 3A,B,E,F). Nevertheless, the calculated TRAMs, which are sensitive to subtle BBBd, reveled significant BBBd which increased with the treatment voltage and with the number of pulses. The TRAMs showed contrast agent accumulation (depicted red) mainly in the cortical region, as predicted by the simulation (Figure 3G-H). The average volume of BBBd found for the sham group (0 V) was $6.08 \pm 3.09 \mathrm{~mm}^{3}$ and the average intensity increase was $6.76 \% \pm 0.2 \%$. The pixels determined to represent BBBd by our analysis algorithm were sporadic pixels scattered in the brain resulting from noise. The average BBBd volume of the minimal treatment group $(100$ pulses at $100 \mathrm{~V})$ was significantly larger than that of the sham group $\left(39.10 \pm 9.66 \mathrm{~mm}^{3}\right.$, $p<0.02)$ and the disrupted volume was concentrated mainly in the cortex of the mice, as can be seen in Figure 3. The increase in signal intensity was also significantly higher than that of the sham group $(13.73 \pm 4.09, p<0.009)$. These results suggest that subtle BBBd was obtained when applying 100 pulses at $100 \mathrm{~V}$.

Table 2. Results of linear regression analysis for the three brain regions.

\begin{tabular}{cccccc}
\hline Brain Region & $\mathbf{r}^{\mathbf{2}}$ & Significance & Prediction Equation & Fold Increase 0-100 & Fold Increase 0-200 \\
\hline Cortex & 0.54 & 0.006 & $\mathrm{BBBd}(\mathrm{V})=0.008 \mathrm{~V}+0.8$ & 3.18 & 3.66 \\
Cerebellum & 0.81 & 0.0002 & $\mathrm{BBBd}(\mathrm{V})=0.004+0.40$ & 1.63 & 3.05 \\
Rest of brain & 0.67 & 0.001 & $\mathrm{BBBd}(\mathrm{V})=0.004+0.53$ & 1.85 & 2.22 \\
\hline
\end{tabular}
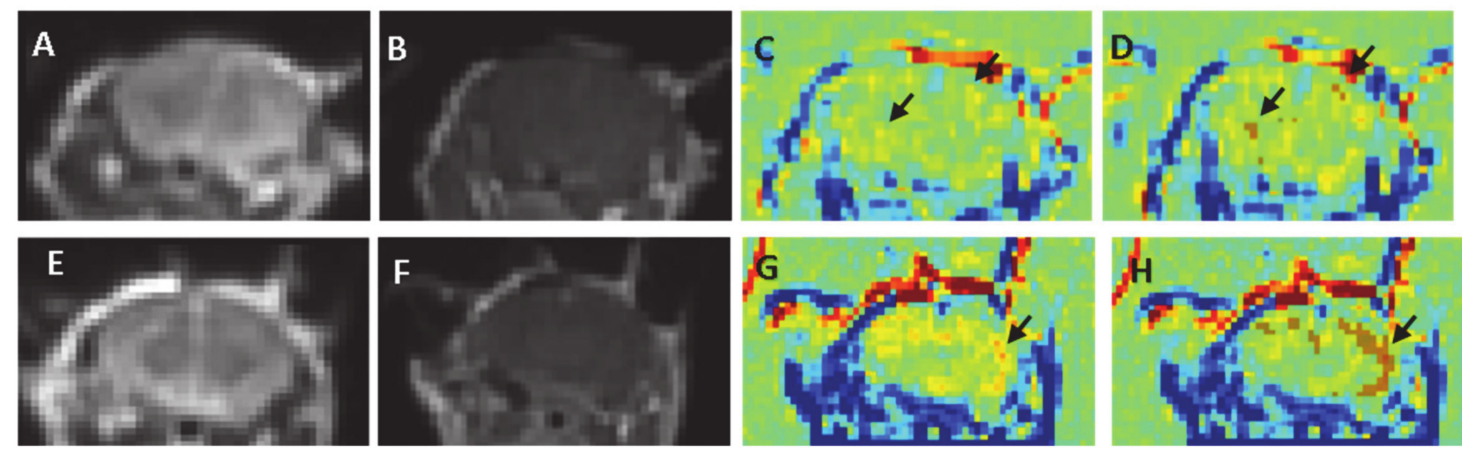

Figure 3. MRIs of a sham mouse and a mouse from the $100 \mathrm{~V}, 100$ pulses group (A-D). Sham, (E,F). $100 \mathrm{~V}, 100$ pulses $(\mathbf{A})+(\mathbf{D})$. Table 2. MRIs showing no signs of damage or edema $(\mathbf{B})+(\mathbf{F})$. Contrast-enhanced T1-MRI showing no visible enhancement in the brain parenchyma. (C) + (G). TRAMs calculated by subtracting the first T1-MRI from the $30 \mathrm{~min}$ T1-MRI. No clusters of contrast accumulation are visible in the sham mouse ((C), arrow shows scattered pixels), while contrast accumulation is visible in the cortex (arrow) of the treated mouse $(\mathbf{G}) .(\mathbf{D})+(\mathbf{H})$ The results of the analysis algorithm showing random small clusters in the sham brain (brown pixels) and significant BBBd clusters (mainly in the cortex) in the treated mouse.

\subsection{Dependency of BBBd on the Applied Voltage}

The dependency of BBBd volume and intensity on the applied voltage was studied in mice treated with 100 pulses at 100, 200 and $300 \mathrm{~V}$ (Figure 4). A linear regression was 
calculated to predict the effect of the applied voltage on the extent of BBBd. A significant regression equation was found $\left(\mathrm{F}(1,19)=85.60, p<1.8 \times 10^{-8}\right)$ with $\mathrm{r}^{2}=0.82$. The results suggest that the applied voltage accounted for $82 \%$ of the variation in BBBd volume, a large size effect according to Cohen [38]. The prediction equation was $\mathrm{BBBd}(\mathrm{V})=0.733 \mathrm{~V}-22.41$, where $\mathrm{V}$ is the applied voltage. The results demonstrated a $336.7 \%$ increase in the average BBBd volume when the voltage was increased from 0 to $300 \mathrm{~V}$, suggesting strong dependence on the voltage (Figure 5A). The dependency of BBBd intensity (increase in signal intensity over time) on the applied voltage was also studied using liner regression. BBBd intensity increased by $216.2 \%$ (from $6.76 \% \pm 0.20 \%$ to $14.63 \% \pm 1.75 \%$ ) when the voltage was increased from 0 to $300 \mathrm{~V}$.

A

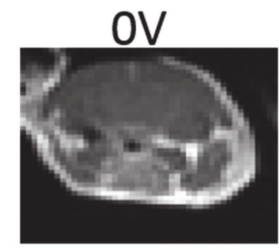

B

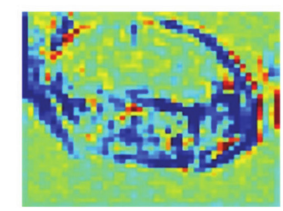

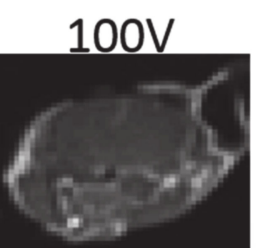

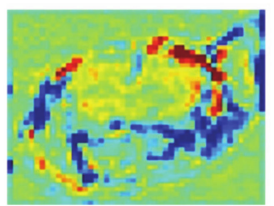

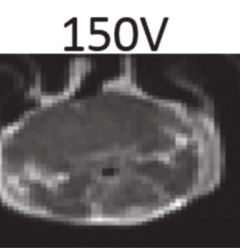

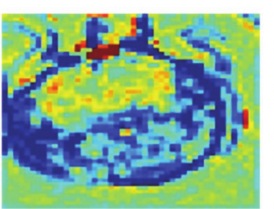

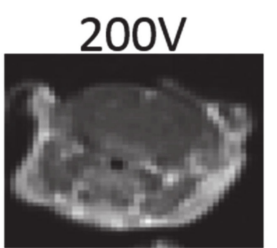

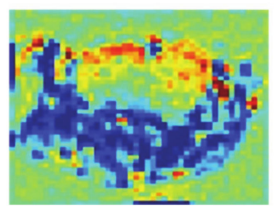

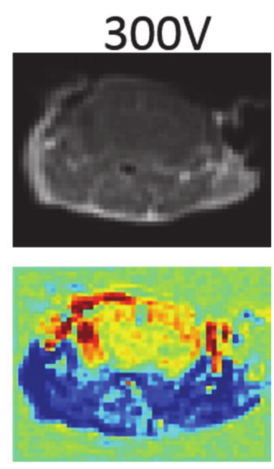

Figure 4. Examples of T1-MRIs and their equivalent $30 \mathrm{~min}$ TRAMs for different treatment voltages. (A) T1-MRI obtained immediately post-contrast injection for sham $(0 \mathrm{~V})$ and for mice treated with 100 pulses at 100-300 V. No enhancement is visible in the brain parenchyma (B). TRAMs calculated by subtracting the first T1-MRI post-contrast injection from the $30 \mathrm{~min}$ T1-MRI, showing increased BBBd volumes (depicted yellow/red, mainly in the cortex) with increased treatment voltage.
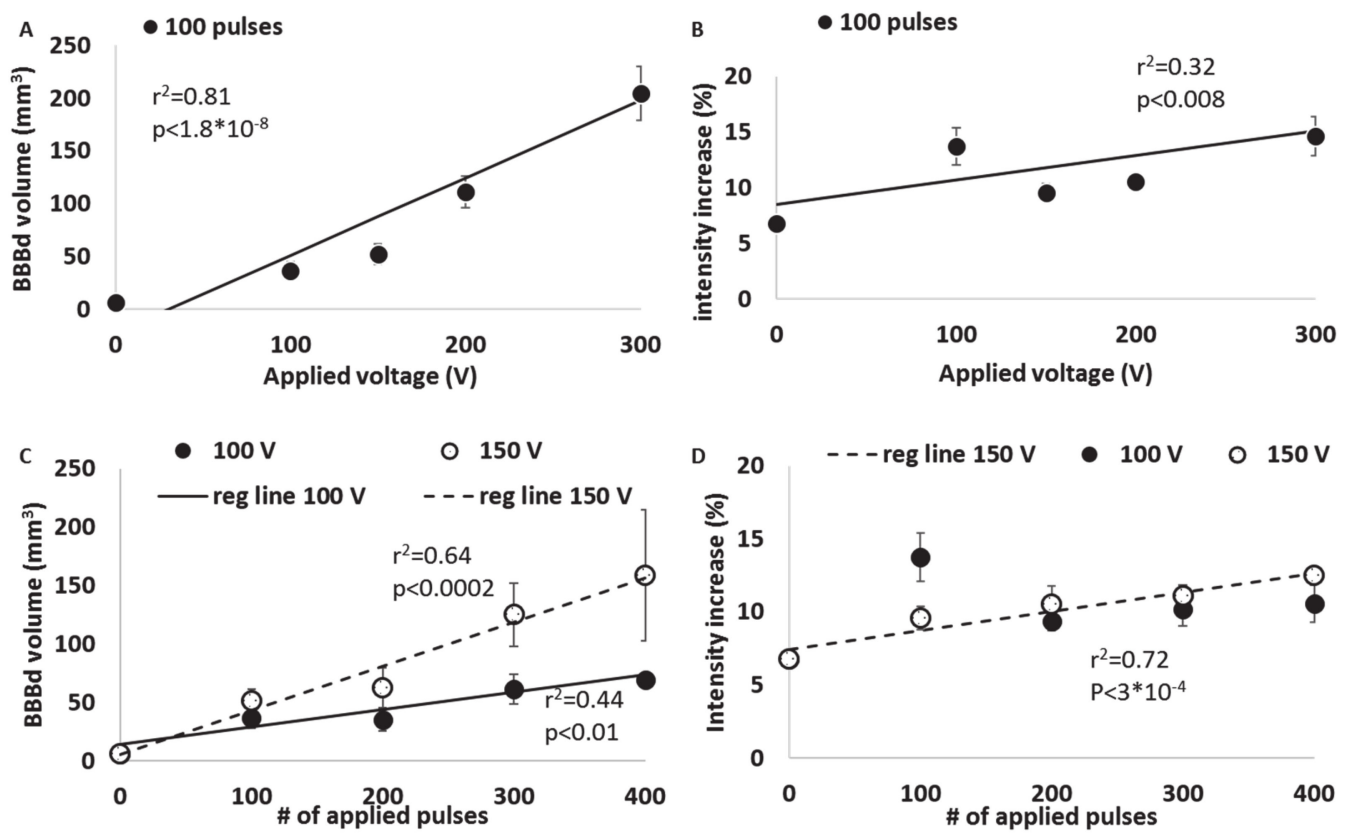

Figure 5. Experimental data and linear regression equation lines describing the dependency of BBBd volume and intensity on the treatment parameters. (A) BBBd volume as a function of the applied voltage for 100 pulses. (B) BBBd intensity increase as a function of the applied voltage. (C). BBBd volume as a function of the number of pulses at 100 and $150 \mathrm{~V}$. (D) BBBd intensity increase as a function of the number of pulses at 100 and $150 \mathrm{~V}$.

A significant regression equation was also found $\mathrm{F}(1,19)=8.76, p<0.008)$ with $\mathrm{r}^{2}=0.32$, suggesting the applied voltage accounted for only $32 \%$ of the variation in BBBd intensity. This result is still considered a large effect size according to Cohen, though 
the results suggest that the relationship between the two variables is weaker than the relationship between the applied voltage and the BBBd volume. The prediction equation was $\operatorname{BBBd}(\mathrm{V})=0.022 \mathrm{~V}-8.47$, where $\mathrm{V}$ is the applied voltage (Figure $5 \mathrm{~B}$ ).

\subsection{Dependency of BBBd on the Number of Pulses}

Linear regressions were calculated to predict the effect of the number of applied pulses on BBBd volume and intensity. Mice were treated using 100, 200, 300 or 400 pulses with applied voltage of either 100 or $150 \mathrm{~V}$. The results demonstrate a $114 \%$ and $131 \%$ increase in the average volume when the number of pulses was increased from 0 to 300 pulses in the 100 and $150 \mathrm{~V}$ treatment groups, respectively. For both 100 and $150 \mathrm{~V}$, significant regression equations were found $(\mathrm{F}(1,20)=15.50, p<0.01)$ with an $\mathrm{r}^{2}=0.44$ for $100 \mathrm{~V}$ and $(\mathrm{F}(1,14)=24.87, p<0.0002)$ with an $\mathrm{r}^{2}=0.64$ for $150 \mathrm{~V}$. The results suggest that the applied voltage accounted for $44 \%$ of the variation in BBBd volume in the $100 \mathrm{~V}$ groups and $64 \%$ in the $150 \mathrm{~V}$ groups. Although both effects are considered large, the effect was greater when a higher voltage was applied. The prediction equations were $\mathrm{BBBd}(\mathrm{P})=0.15 \mathrm{P}+14.09$ and $\operatorname{BBBd}(\mathrm{P})=0.38 \mathrm{P}+5.24$ for 100 and $150 \mathrm{~V}$, respectively, where $\mathrm{P}$ is the number of applied pulses. The larger coefficient of the $150 \mathrm{~V}$ prediction equation also indicates that increasing the number of pulses at higher voltage has a larger effect (Figure 5C). BBBd intensity increased by $156.20 \%$ and $185.06 \%$ when the number of pulses was increased from 0 to 400 for 100 and $150 \mathrm{~V}$, respectively. Nevertheless, the linear regression for the $100 \mathrm{~V}$ groups was not significant $(\mathrm{F}(1,20)=0.15, p<0.7)$ with $\mathrm{r}^{2}=0.07$. This result suggests that although the signal intensity increased significantly between 0 and 100 pulses as described above, increasing the number of pulses did not increase the BBBd intensity. Nevertheless, for the $150 \mathrm{~V}$ groups, a significant regression equation was found between the number of applied pulses and $\mathrm{BBBd}$ intensity $\left(\mathrm{F}(1,14)=59.7, p<3 \times 10^{-4}\right)$ with $\mathrm{r}^{2}=0.72$. These results suggest that the number of pulses accounted for $72 \%$ of the variation in BBBd intensity in the $150 \mathrm{~V}$ groups (Figure 5D). The results of the regression analysis of BBBd intensity are in accordance with the results of BBBd volume regression analysis, suggesting that when the voltage is increased, the effect of the number of pulses increases as well.

\subsection{Safety MRI Experiments}

In order to evaluate late treatment effects, six mice underwent MRI $48 \mathrm{~h}$ post-treatment. No enhancement was observed on the contrast-enhanced T1- MRIs nor hyper-intense regions on the T2-MRIs that might suggest BBBd, edema or tissue damage. No hypointense regions were observed on either the GE images or the SWAN images, suggesting no bleedings occurred. Figure 6 shows representative brain slices of a mouse treated with 100 pulses at $300 \mathrm{~V}$ (the highest applied voltage).
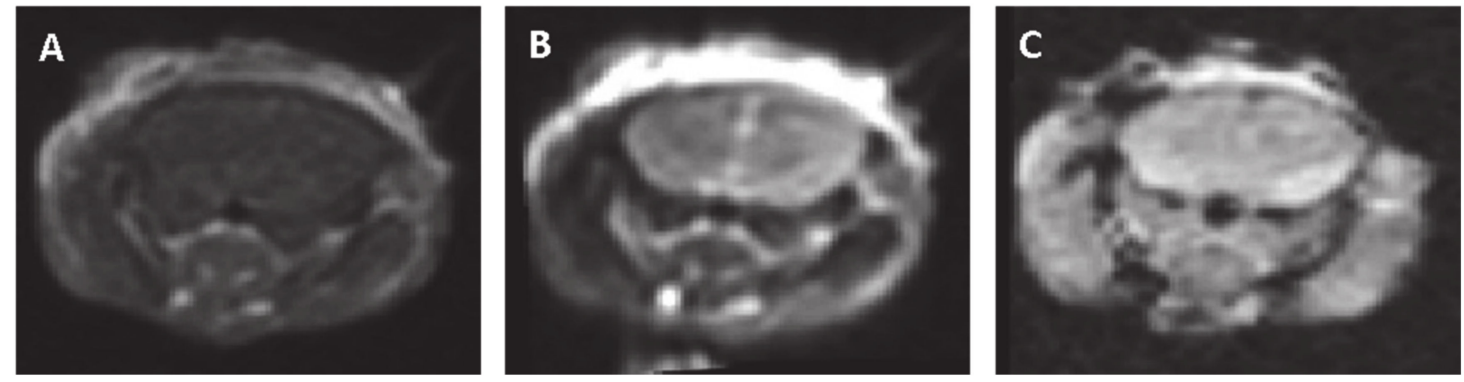

Figure 6. Representative MRI slices obtained $48 \mathrm{~h}$ post-pulsed electrical fields (PEFs) application (100 pulses at $300 \mathrm{~V})$ (A). Contrast-enhanced T1-MRI. (B) T2-MRI. (C) Gradient echo (GE) MRI.

\subsection{Evans Blue Extravasation Experiments}

EB extravasation post-PEFs application was visible to the naked eye compared to the sham brains (Figure 7A-C). Following extraction, the brains were processed for quantifi- 
cation of the extravasation. The results are presented in img EB per $\mathrm{g}$ brain. Similarly to the MRI experiments, three linear regressions were conducted, one for each part of the brain-cortex, cerebellum and the remaining part of the brain. Significant regression equations were found for all three regions, supporting the results of the MRI experiments. The results of the regression are summarized in Table 2 and in Figure 7D. These results clearly demonstrate that the BBB was disrupted as EB does not penetrate the intact BBB.
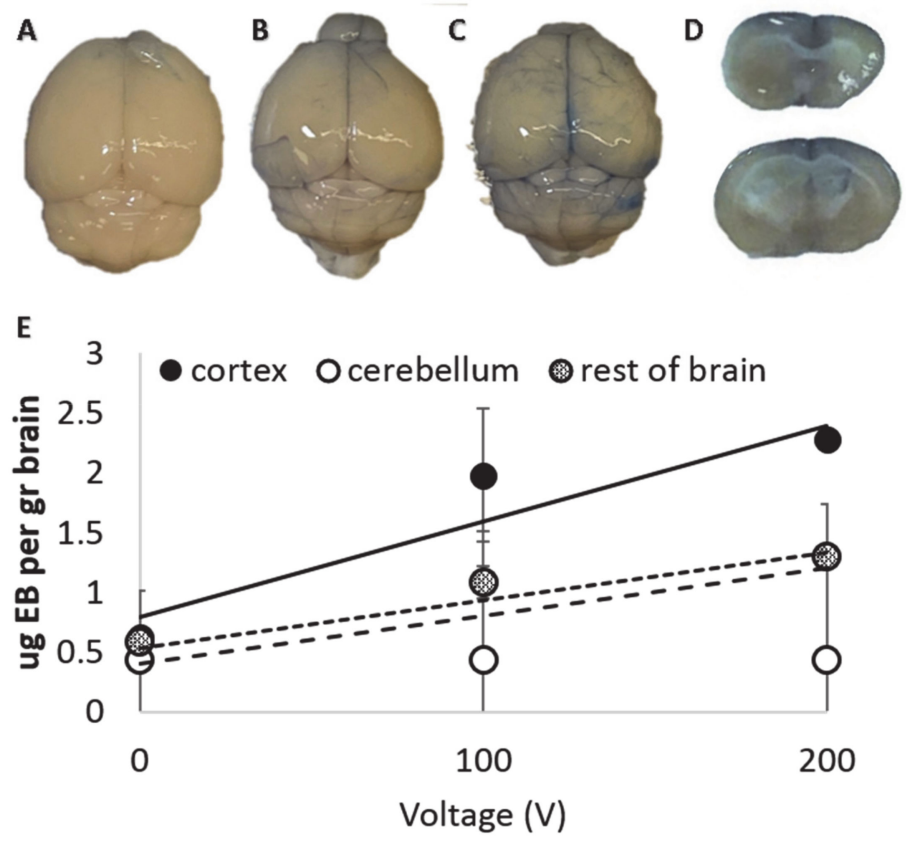

Figure 7. Results of the Evans blue (EB) extravasation experiments. (A) A brain of a mouse treated with the sham protocol after 20 min perfusion. (B) A brain of a mouse treated with 100 pulses at $100 \mathrm{~V}$. (C) A brain of a mouse treated with 100 pulses at $200 \mathrm{~V}$. (D) Coronal slices of a mouse treated with 100 (top) and $200 \mathrm{~V}$ (bottom). EB is seen mainly in the cortex but can also be seen in the striatum. (E) The amount of EB found in the tissue as a function of the applied voltage for the different brain regions.

The results corroborate the results of the MRIs demonstrating stronger BBBd in the cortex than in the rest of the brain. They further demonstrate the treatment ability to enable transport of not only small molecules such as Gd-DOTA, but also macromolecules, as EB binds serum albumin with high affinity [39].

\subsection{Numerical Model}

The electric field distribution for the different voltages was calculated using the finite elements model. The results were extracted to Matlab and interpolated to match the MRI resolution. The maximal electric field was extracted for each voltage. The relationship between the applied voltage and maximal electric field in the brain can be described using a linear function:

$$
\mathrm{EF}_{\max }=0.624 \mathrm{~V} 624 \mathrm{~V}
$$

where $\mathrm{EF}_{\max }$ is the maximal electric field in the brain and $\mathrm{V}$ is the applied voltage. The brain slices with the maximal electric field values are presented in Figure 8A-D. For each applied voltage, the highest electric field values were found in the cortex. This result is in accordance with the MRI and EB experiments demonstrating the stronger treatment effect was found in the cortex. The finite elements model was used to calculate the change in brain temperature since an increase of $>1{ }^{\circ} \mathrm{C}$ in brain temperature can induce BBBd [40]. There are two mechanisms that may cause a temperature elevation. The first is the Joule heating induced by the electric field in the brain and the second is heat conduction from the less conductive tissues such as the bone and dura. The maximal temperature was determined 
to be $37.17^{\circ} \mathrm{C}$, i.e., a temperature increase of $0.37^{\circ} \mathrm{C}$. This temperature was achieved after 400 pulses at $150 \mathrm{~V}$. Treatment with 100 pulses at $300 \mathrm{~V}$ increased the temperature from 36.8 to only $37.06{ }^{\circ} \mathrm{C}$. The brain slices with the highest temperature for 100 pulses are shown in Figure $8 \mathrm{E}-\mathrm{H}$.
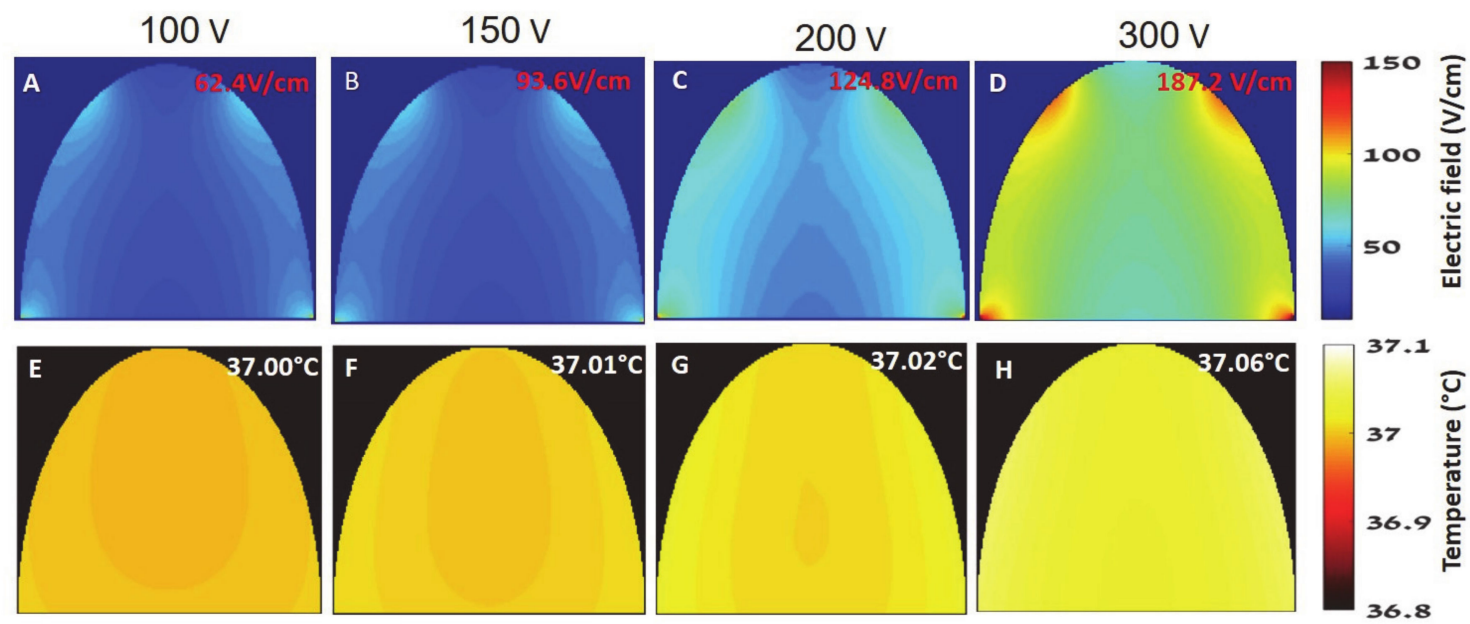

Figure 8. Electric field and temperature distributions after 100 pulses as calculated from the finite elements simulation (A-D). Electric field distribution (E-H). Temperature distribution. (A) + (E) 100 V. (B) + (F) 150 V. (C) + (G) 200 V. (D) + (H) $300 \mathrm{~V}$.

\section{Discussion}

The BBB presents a significant obstacle for treating brain diseases such as neurodegenerative disorders and brain tumors [41]. Thus, means to safely disrupt the BBB are of urgent need. We recently demonstrated that short treatments with low PEFs can induce transient BBBd in vitro [23].

Here, our objective was to demonstrate the feasibility of inducing similar BBBd in vivo by non-invasive (intact skull) low PEFs and study the effects of different treatment parameters (applied voltage and number of pulses) on the extent of BBBd. The study was performed in mice. We found that application of 100-400 pulses at 100-300 V can induce subtle BBBd, undetectable by conventional T1-MRI but clearly depicted using the TRAMs. Subtle BBBd using the same treatment protocols with similar dependence on the treatment parameters was also found in the EB experiments.

The TRAMs depicted continuous contrast accumulation over at least $30 \mathrm{~min}$ postcontrast injection. The advantage of using delayed-contrast MRI over standard T1-MRI is increased sensitivity to subtle BBBd undetected by conventional MRI techniques $[20,24]$ and the additional information it provides such as time to peak and wash-in/wash-out rates. This additional information may be used for better characterization of the extent of disruption and for determination of the optimal time window for systemic therapeutics administration. Here, we used the maximal intensity increase and the goodness of fit parameters in order to determine BBBd volumes and levels, but in the future, more parameters can be derived from the TRAMs data.

The relationship between the applied voltage and BBBd volume/intensity calculated from the MRIs was studied using linear regressions. The MRI experiments reveled a strong linear relationship between the applied voltage and BBBd volume and a weaker but still significant linear relationship between the applied voltage and BBBd intensity. The EB experiments showed a similar relationship with the amount of dye per gram of brain. Therefore, an important conclusion from the study is that increasing the applied voltage can increase both BBBd volumes and intensities, thus enabling higher therapeutic doses to enter the brain. 
Increasing the number of pulses also increased the BBBd volume but to a lesser extent as demonstrated by the smaller coefficient of the prediction equation. Still, the dependence on the number of pulses was found to be stronger when increasing the treatment voltage from 100 to $150 \mathrm{~V}$, suggesting that the relationship is more complex. In the future, a model incorporating both the applied voltage and the number of pulses for simulating low PEFsinduced BBBd should be considered. This can be conducted, for example, by using the Peleg-Fermi model for cell kill probability as a function of treatment voltage and number of pulses, as we have previously conducted when extending the model for EP-induced BBBd $[19,42]$.

The MRI experiments demonstrated the feasibility for passage of small molecules (Gd-DOTA (753.9 Da) across the BBB and that BBBd extent increased with the applied voltage and number of pulses. The results of the EB extravasation experiments not only support the conclusions from the MRI experiments, but also demonstrate that low PEFs induced BBBd, enabling the passage of macromolecules, as EB binds to serum albumin $(66 \mathrm{kDa})$ in the blood.

Both the MRI and the EB extravasation experiments found increased BBBd in the cortex compared to the rest of the brain. These results are in accordance with the finite elements model results showing that the highest electric field was reached in the cortex. Thus, it may be hypothesized that the main parameter affecting the extent of BBBd is the electric field. The maximal electric field in the brain as calculated by the finite elements model for $100 \mathrm{~V}$ was $62.4 \mathrm{~V} / \mathrm{cm}$ and reached $187.2 \mathrm{~V} / \mathrm{cm}$ for $300 \mathrm{~V}$. It was previously demonstrated by us and others that EP can induce BBBd with electric fields thresholds ranging between 500 and $700 \mathrm{~V} / \mathrm{cm}[17,19]$. Recently, Lorenzo et al. [21] demonstrated that H-FRE also induces BBBd, at a lower threshold $(113 \mathrm{~V} / \mathrm{cm})$, when using two needle electrodes inserted into the brain parenchyma with a voltage to distance ratio of $600 \mathrm{~V} / \mathrm{cm}$. Although determining the electric field threshold for BBBd was beyond the scope of this study, the maximal electric fields induced in the brain using our closed cranium setup were significantly smaller $(62.4 \mathrm{~V} / \mathrm{cm}$ for $100 \mathrm{~V})$ by an order of magnitude than those previously described in the literature as the threshold for EP and by a factor of 2 than the H-FRE threshold.

One explanation for these differences can be the increased sensitivity of the TRAMs to subtle BBBd compared to conventional MRI. Another explanation may be that the mechanism for inducing BBBd using low PEFs is different than EP. In addition, those EP and H-FRE experiments were conducted using either one or two needle electrodes inserted into the brain through burr holes drilled in the skull. This method of inducing BBBd requires induction of high electric fields in the brain which may cause, in addition to BBBd, tissue damage in smaller volumes. Even if no irreversible EP occurs, damage along the electrodes path is expected [21].

On top of that, exposure to high electric fields can induce significant vasodilatation which can, by itself, induce BBBd but also increase wash-out rates of therapeutic agents [20], thus diminishing the efficacy of the disruption. We previously applied TRAMs for studying the effects of point source EP (EP induced by a minimally invasive setup consisting of one partially insulated intracranial needle and one external surface electrode) $[12,17,18,21]$. In the point source study, EP-induced BBBd appeared as an enhancing region on standard T1-MRI and was depicted as blue in the TRAMs (negative signal), consistent with vasodilatation and fast contrast clearance rates. The enhancing region was surrounded by a thin red rim in the TRAMs, representing contrast accumulation.

In the current study, the subtle BBBd induced by low PEFs did not show any enhancement on standard T1-MRI and was depicted red in the TRAMs. This suggests that EP-induced BBBd is depicted differently on TRAMs than low PEFs-induced subtle BBBd. Although the mechanism of action of low PEFs-induced BBBd remains unclear, in vitro studies demonstrated that EP is not part of the mechanism of action. It has been suggested that low PEFs induce BBBd via the paracellular pathway, by affecting the tight 
junctions and adherent junctions proteins $[23,43]$, although the exact mechanism of action remains unclear.

The results of the MRIs acquired $48 \mathrm{~h}$ post-treatment reveal no signs of hemorrhage, edema or damage. Nevertheless, these results do not rule out microscale damage to the tissue or microcirculation which may be below the sensitivity of the MRI. Thus, a comprehensive histological study, using brains extracted at different time points posttreatment, is needed to evaluate both early and late possible tissue changes and damage.

It is important to note that we address our results as demonstrating the feasibility for inducing BBBd non-invasively since the treatment was performed extracranially, i.e., without opening the skull, but was not non-invasive. The skin of the mice was cut open to allow the electrodes to be in direct contact with the intact skull. This was conducted in order to reduce the loss in the electric field strength in the non-conducting skin and fur due to technical limitations of our experimental system. Still, it is possible to achieve similar electric fields in the brain without opening the skin by, for example, increasing the applied voltage or changing the electrode setup. Further research and development are needed to achieve a completely non-invasive treatment protocol suitable for clinical application.

Non-invasiveness is a major advantage of low PEFs-induced BBBd. An additional advantage is the short treatment duration (only several minutes). In the future, these advantages may enable the development of safe treatment protocols combined with systemic drug administration for CNS disorders that can be repeated as needed.

\section{Conclusions}

Our results demonstrate the feasibility of applying low PEFs non-invasively for inducing subtle yet significant BBBd. A significant linear relationship was found between the extent of BBBd and the applied voltage and number of pulses. EB extravasation was also found in the brain post-low PEFs application, suggesting that disruption was not limited to small molecules. Our results suggest significantly lower electric field thresholds for BBBd than previously reported and they present the first proof of concept for a non-invasive application of PEFs-induced BBBd. As the treatment is rapid and may be applied in a non-invasive manner, it may be used repeatedly in parallel to systemic drug administration for efficient delivery of therapeutic agents into the brain. In the future, the efficacy of combining non-invasive low PEFs with drug therapy for CNS disorders such as neurodegenerative diseases and brain tumors should be evaluated.

\section{Patents}

Pending PCT \#WO2019175871A1 titled "method for changing blood brain barrier permeability" was filed based on the study results.

Author Contributions: All authors contributed to the manuscript. Specific contributions: Conceptualization, S.S., I.C. and Y.M.; methodology, S.S., I.C., Y.M., S.L.-Z. and D.L.; investigation, Y.B., S.S., D.L., D.D., D.A. and S.L.-Z.; software, S.S., D.L. and D.D.; formal analysis, S.S., D.D., D.A., I.D.F. and Y.B.; writing—original draft preparation, S.S.; writing—review and editing, S.S., I.C., I.D.F. and Y.M.; funding acquisition, Y.M., I.C. and S.S. All authors have read and agreed to the published version of the manuscript.

Funding: The study was supported by the Israel Science foundation (Grant 1922/18).

Institutional Review Board Statement: The study was conducted according to the guidelines of the Declaration of Helsinki, and approved by Sheba Medical Center and the Animal Care and Use Committee of Sheba Medical Center approved the protocol (\#126020 9/9/20 and \#113918 25/6/18).

Informed Consent Statement: Not applicable.

Data Availability Statement: The data presented in this study including full MRI scans are available on request from the corresponding author.

Conflicts of Interest: Authors S.S., Y.B., D.L., D.D., Y.M. and I.C. are inventors. 


\section{References}

1. Dong, X. Current Strategies for Brain Drug Delivery. Theranostics 2018, 8, 1481-1493. [CrossRef]

2. Hashimoto, Y.; Campbell, M. Tight junction modulation at the blood-brain barrier: Current and future perspectives. Biochim. Biophys. Acta Biomembr. 2020, 1862, 183298. [CrossRef]

3. Patel, M.M.; Patel, B.M. Crossing the Blood-Brain Barrier: Recent Advances in Drug Delivery to the Brain. CNS Drugs 2017, 31, 109-133. [CrossRef]

4. Alexander, A.; Agrawal, M.; Uddin, A.; Siddique, S.; Shehata, A.M.; Shaker, M.A.; Rahman, S.A.U.; Abdul, M.I.M.; Shaker, M.A. Recent expansions of novel strategies towards the drug targeting into the brain. Int. J. Nanomed. 2019, 14, 5895-5909. [CrossRef]

5. Rossmeisl, J.H.; Herpai, D.; Quigley, M.; Cecere, T.E.; Robertson, J.L.; D’Agostino, R.B.; Debinski, W. Phase I trial of convectionenhanced delivery of IL13RA2 and EPHA2 receptor targeted cytotoxins in dogs with spontaneous intracranial gliomas. Neuro. Oncol. 2020. [CrossRef]

6. Heiss, J.D.; Lungu, C.; Hammoud, D.A.; Herscovitch, P.; Ehrlich, D.J.; Argersinger, D.P.; Bankiewicz, K.S. Trial of magnetic resonance-guided putaminal gene therapy for advanced Parkinson's disease. Mov. Disord. 2019, 34, 1073-1078. [CrossRef]

7. Wang, H.-L.; Kuo, E.Y.; Lai, T.W. Vascular delivery of intraperitoneal Evans blue dye into the blood-brain barrier-intact and disrupted rat brains. NeuroReport 2018, 29, 924-931. [CrossRef] [PubMed]

8. Beccaria, K.; Sabbagh, A.; De Groot, J.; Canney, M.; Carpentier, A.; Heimberger, A.B. Blood-brain barrier opening with low intensity pulsed ultrasound for immune modulation and immune therapeutic delivery to CNS tumors. J. Neuro Oncol. 2020, 1-9. [CrossRef] [PubMed]

9. Rodriguez, A.; Tatter, S.B.; Debinski, W. Neurosurgical Techniques for Disruption of the Blood-Brain Barrier for Glioblastoma Treatment. Pharmaceutics 2015, 7, 175-187. [CrossRef] [PubMed]

10. Yarmush, M.L.; Golberg, A.; Serša, G.; Kotnik, T.; Miklavčič, D. Electroporation-based technologies for medicine: principles, applications, and challenges. Biomed. Eng. 2014, 16, 295-320. [CrossRef] [PubMed]

11. Sharabi, S.; Guez, D.; Daniels, D.; Cooper, I.; Atrakchi, D.; Liraz-Zaltsman, S.; Last, D.; Mardor, Y. The application of point source electroporation and chemotherapy for the treatment of glioma: A randomized controlled rat study. Sci. Rep. 2020, 10. [CrossRef] [PubMed]

12. Latouche, E.L.; Arena, C.B.; Ivey, J.W.; Garcia, P.A.; Pancotto, T.E.; Pavlisko, N.; Verbridge, S.S.; Davalos, R.V.; Rossmeisl, J.H. High-Frequency Irreversible Electroporation for Intracranial Meningioma: A Feasibility Study in a Spontaneous Canine Tumor Model. Technol. Cancer Res. Treat. 2018, 17, 1533033818785285. [CrossRef] [PubMed]

13. Garcia, P.A.; Kos, B.; Rossmeisl, J.H.; Pavliha, D.; Miklavcic, D.; Davalos, R.V. Predictive therapeutic planning for irreversible electroporation treatment of spontaneous malignant glioma. Med. Phys. 2017, 44, 4968-4980. [CrossRef] [PubMed]

14. O'Neill, C.; Hayat, T.; Hamm, J.; Healey, M.; Zheng, Q.; Li, Y.; Martin, R.C.G. A phase $1 \mathrm{~b}$ trial of concurrent immunotherapy and irreversible electroporation in the treatment of locally advanced pancreatic adenocarcinoma. Surgery 2020, 168, 610-616. [CrossRef]

15. Frandsen, S.K.; Vissing, M.; Gehl, J. A Comprehensive Review of Calcium Electroporation-A Novel Cancer Treatment Modality. Cancers 2020, 12, 290. [CrossRef] [PubMed]

16. Wichtowski, M.; Murawa, D.; Czarnecki, R.; Piechocki, J.; Nowecki, Z.; Witkiewicz, W. Electrochemotherapy in the Treatment of Breast Cancer Metastasis to the Skin and Subcutaneous Tissue-Multicenter Experience. Oncol. Res. Treat. 2018, 42, 47-51. [CrossRef]

17. Garcia, P.A.; Rossmeisl, J.H.; Robertson, J.L.; Olson, J.D.; Johnson, A.J.; Ellis, T.L.; Davalos, R.V. 7.0-T Magnetic Resonance Imaging Characterization of Acute Blood-Brain-Barrier Disruption Achieved with Intracranial Irreversible Electroporation. PLoS ONE 2012, 7, e50482. [CrossRef]

18. Sharabi, S.; Last, D.; Guez, D.; Daniels, D.; Hjouj, M.I.; Salomon, S.; Maor, E.; Mardor, Y. Dynamic effects of point source electroporation on the rat brain tissue. Bioelectrochemistry 2014, 99, 30-39. [CrossRef]

19. Sharabi, S.; Kos, B.; Last, D.; Guez, D.; Daniels, D.; Harnof, S.; Mardor, Y.; Miklavcic, D. A statistical model describing combined irreversible electroporation and electroporation-induced blood-brain barrier disruption. Radiol. Oncol. 2016, 50, 28-38. [CrossRef]

20. Sharabi, S.; Last, D.; Daniels, D.; Zaltsman, S.L.; Mardor, Y. The effects of point-source electroporation on the blood-brain barrier and brain vasculature in rats: An MRI and histology study. Bioelectrochemistry 2020, 134, 107521. [CrossRef]

21. Lorenzo, M.F.; Thomas, S.C.; Kani, Y.; Hinckley, J.; Lee, M.; Adler, J.; Verbridge, S.S.; Hsu, F.-C.; Robertson, J.L.; Davalos, R.V.; et al. Temporal Characterization of Blood-Brain Barrier Disruption with High-Frequency Electroporation. Cancers 2019, $11,1850$. [CrossRef] [PubMed]

22. Hjouj, M.; Last, D.; Guez, D.; Daniels, D.; Sharabi, S.; Lavee, J.; Rubinsky, B.; Mardor, Y. MRI Study on Reversible and Irreversible Electroporation Induced Blood Brain Barrier Disruption. PLoS ONE 2012, 7, e42817. [CrossRef] [PubMed]

23. Sharabi, S.; Bresler, Y.; Ravid, O.; Shemesh, C.; Atrakchi, D.; Schnaider-Beeri, M.; Gosselet, F.; Dehouck, L.; Last, D.; Guez, D.; et al. Transient blood-brain barrier disruption is induced by low pulsed electrical fields in vitro: An analysis of permeability and trans-endothelial electric resistivity. Drug Deliv. 2019, 26, 459-469. [CrossRef] [PubMed]

24. Zach, L.; Guez, D.; Last, D.; Daniels, D.; Grober, Y.; Nissim, O.; Hoffmann, C.; Nass, D.; Talianski, A.; Spiegelmann, R.; et al. Delayed contrast extravasation MRI: A new paradigm in neuro-oncology. Neuro-Oncology 2015, 17, 457-465. [CrossRef] [PubMed] 
25. Israeli, D.; Tanne, D.; Daniels, D.; Last, D.; Shneor, R.; Guez, D.; Landau, E.; Roth, Y.; Ocherashvilli, A.; Bakon, M.; et al. The Application of MRI for Depiction of Subtle Blood Brain Barrier Disruption in Stroke. Int. J. Biol. Sci. 2011, 7, 1-8. [CrossRef] [PubMed]

26. Tofts, P.S.; Kermode, A.G. Measurement of the blood-brain barrier permeability and leakage space using dynamic MR imaging. 1. Fundamental concepts. Magn. Reson. Med. 1991, 17, 357-367. [CrossRef]

27. Khalifa, F.; Soliman, A.; El-Baz, A.; El-Ghar, M.A.; El-Diasty, T.; Gimel'Farb, G.; Ouseph, R.; Dwyer, A.C. Models and methods for analyzing DCE-MRI: A review. Med. Phys. 2014, 41, 124301. [CrossRef]

28. Donaldson, S.B.; West, C.M.L.; Davidson, S.E.; Carrington, B.M.; Hutchison, G.; Jones, A.P.; Sourbron, S.P.; Buckley, D.L. A comparison of tracer kinetic models for T 1 -weighted dynamic contrast-enhanced MRI: Application in carcinoma of the cervix. Magn. Reson. Med. 2010, 63, 691-700. [CrossRef]

29. Goldim, M.P.D.S.; Della Giustina, A.; Petronilho, F. Using Evans Blue Dye to Determine Blood-Brain Barrier Integrity in Rodents. Curr. Protoc. Immunol. 2019, 126, e83. [CrossRef]

30. Wang, H.-L.; Lai, T.W. Optimization of Evans blue quantitation in limited rat tissue samples. Sci. Rep. 2014, 4, 6588. [CrossRef]

31. Gentilal, N.; Miranda, P.C. Heat transfer during TTFields treatment: Influence of the uncertainty of the electric and thermal parameters on the predicted temperature distribution. Comput. Methods Programs Biomed. 2020, 196, 105706. [CrossRef] [PubMed]

32. Nagel, S.J.; Reddy, C.G.; Frizon, L.A.; Chardon, M.K.; Holland, M.T.; Machado, A.G.; Gillies, G.T.; Howard, M.A.; Wilson, S. Spinal dura mater: Biophysical characteristics relevant to medical device development. J. Med. Eng. Technol. 2018, 42, 128-139. [CrossRef] [PubMed]

33. Elwassif, M.M.; Kong, Q.; Vazquez, M.; Bikson, M. Bio-heat transfer model of deep brain stimulation-induced temperature changes. J. Neural. Eng. 2006, 3, 306-315. [CrossRef]

34. Sel, D.; Lebar, A.M.; Miklavcic, D. Feasibility of Employing Model-Based Optimization of Pulse Amplitude and Electrode Distance for Effective Tumor Electropermeabilization. IEEE Trans. Biomed. Eng. 2007, 54, 773-781. [CrossRef] [PubMed]

35. Ramon, C.; Garguilo, P.; Fridgeirsson, E.A.; Ehaueisen, J. Changes in scalp potentials and spatial smoothing effects of inclusion of dura layer in human head models for EEG simulations. Front. Neuroeng. 2014, 7, 32. [CrossRef]

36. Garcia, P.A.; Pearce, J.A.; Davalos, R.V. A Comparison Between the Pulsed and Duty Cycle Approaches Used to Capture the Thermal Response of Tissue During Electroporation-Based Therapies. In Proceedings of the ASME 2012 Summer Bioengineering Conference, Parts A and B, Fajardo, PR, USA, 20-23 June 2012.

37. Wong, W.K.; Lachenbruch, P.A. Tutorial in biostatistics. Designing studies for dose response. Stat. Med. 1996, 15, 343-359. [CrossRef]

38. Cohen, J. Statistical Power Analysis for the Behavioral Sciences, 2nd ed.; Routledge: New York, NY, USA, 1988.

39. Hawkins, B.T.; Egleton, R.D. Fluorescence imaging of blood-brain barrier disruption. J. Neurosci. Methods 2006, 151, 262-267. [CrossRef]

40. Kiyatkin, E.A.; Sharma, H.S. Permeability of the blood-brain barrier depends on brain temperature. Neuroscience 2009, 161, 926-939. [CrossRef]

41. Pardridge, W.M. Drug Transport across the Blood-Brain Barrier. Br. J. Pharmacol. 2012, 32, 1959-1972. [CrossRef]

42. Peleg, M. A model of microbial survival after exposure to pulsed electric fields. J. Sci. Food Agric. 1995, 67, 93-99. [CrossRef]

43. Lopez-Quintero, S.V.; Datta, A.; Amaya, R.; Elwassif, M.; Bikson, M.; Tarbell, J.M. DBS-relevant electric fields increase hydraulic conductivity of in vitro endothelial monolayers. J. Neural. Eng. 2010, 7, 16005. [CrossRef] [PubMed] 